\title{
MODELING CHARACTERISTICS OF REAL LOUDSPEAKERS USING VARIOUS ACOUSTIC MODELS: MODAL-DOMAIN APPROACHES
}

\author{
Yonggang $\mathrm{Hu}^{1 *}$, Prasanga N. Samarasinghe ${ }^{1}$, Thushara D. Abhayapala ${ }^{1}$, Glenn Dickins ${ }^{2}$ \\ ${ }^{1}$ Research School of Engineering, Australian National University, Canberra, Australia \\ ${ }^{2}$ Dolby Laboratories, Sydney, Australia
}

\begin{abstract}
The accuracy and perception of soundfields produced by loudspeaker arrays are strongly influenced by the inherent characteristics of the commercial loudspeakers. This paper analyzes such characteristics of loudspeakers by deriving equivalent theoretical models, and by studying their impact on soundfield reproduction. A number of acoustic models are investigated, including plane waves decomposition, point source decomposition and mixed source decomposition. Each proposed model employs three effective sparse decomposition algorithms for optimized solutions, including iteratively reweighted least squares (IRLS), matching pursuit (MP) and least absolute shrinkage and selection operator (LASSO). A successful model shall enable the prediction of the soundfield outside the original recording region. Therefore, we validate the effectiveness of the models by comparing the simulated soundfield with secondary measurements obtained beyond the original area. Experimental results have confirmed that both the plane wave and mixed source model achieve promising performance with respect to the proposed metrics.
\end{abstract}

Index Terms - Loudspeaker modeling, spatial sound reproduction, sparse equivalent source modeling.

\section{INTRODUCTION}

Typically, spatial soundfield reproduction uses an array of loudspeakers to create an immersive soundfield over a predefined spatial region so that listeners within the area can experience a virtual but realistic replication of the original soundfield [1-6]. Such perception can be achieved by controlling the locations and driving signal of the loudspeakers that enclose the spatial region of interest.

A considerable amount of soundfield reproduction systems have been proposed and can be categorized into three types: (i) channel based, (ii) object based, and (iii) model based methods. However, neither channel-based nor objectbased approaches achieve promising reproductions as they focus more on creating an artistic impression of surround sound. Nowadays, soundfield reproductions are often implemented using model based techniques derived from basic wave equations. Among the model based approaches, higher order Ambisonics [7-10] and Wave Field Synthesis [11-14] have become the two most popular techniques in the field of soundfield reproduction for the recent decade. Nevertheless, even

\footnotetext{
* Thanks to Australian Research Council Linkage Grant Projects funding scheme (project no. LP160100379). Yonggang Hu is sponsored by CSC agency for funding.
}

with the effective model based algorithms, accurate spatial soundfield reproductions over space are still to be realized. While a number of factors are causing this, one that needs immediate attention shall be the assumption of omnidirectional behavior of sound sources, which is not the case with commercial loudspeakers. To the best of our knowledge, there are very few techniques known to the authors to characterize or take such consideration into soundfield reproduction systems attempting to be technically accurate and precise. Given commercial loudspeakers are inherently directional with frequency dependence, we aim to model characteristics of loudspeakers using an equivalent source model. In [15], we have presented initial results on this topic by proposing an equivalent plane wave decomposition. However, this modelling is confined to a limited range of frequency and suffers from a slow convergence rate.

This paper continues to conduct further research into this important topic over a wider frequency band. Apart from the plane wave model, we explore an equivalent point source decomposition model with various radii, and a mixed acoustic model that combines both the plane wave and point source decompositions. Since loudspeakers are modeled individually, the resulting incident field at the listening area is inherently sparse, specially in terms of incident direction. Therefore, the aforementioned equivalent source models can be further optimized by exploiting the feature of spatial sparsity. Thus, each acoustic model analyzes a range of sparsity exploitation algorithms [16], including iteratively re-weighted least squares (IRLS), matching pursuit (MP) and least absolute shrinkage and selection operator (LASSO). Finally, extensive experiments are conducted using a moving commercial microphone array to validate the effectiveness of proposed models.

\section{PROBLEM FORMULATION}

In this section, we describe the soundfield produced by a loudspeaker, observed in a listening area, and then formulate the problem to be addressed. Typically, any arbitrary soundfield at a point $\mathbf{x}=(r, \theta, \phi)$ within a spherical listening region of radius $R$ can be decomposed into modal domain [17] by,

$$
P(\boldsymbol{x}, k)=\sum_{n=0}^{N} \sum_{m=-n}^{n} \alpha_{n m}(k) j_{n}(k r) Y_{n m}(\theta, \phi)
$$

where $N=\lceil k R\rceil$ indicates the order of soundfield [7] and $\alpha_{n m}(k)$ represents the spherical harmonics coefficients. The $j_{n}(\cdot)$ stands for spherical Bessel functions and $Y_{n m}(\theta, \phi)$ is the spherical harmonics function with order $n$ and degree $m$.

Traditionally, we assume that the loudspeakers act as an omni-directional point sources $[7,18]$ so that $\alpha_{n m}(k)$ due to 
a loudspeaker located at $\left(r_{s}, \theta_{s}, \phi_{s}\right)$ can be calculated as,

$$
\alpha_{n m}(k)=4 \pi i k h_{n}\left(k r_{s}\right) Y_{n m}\left(\theta_{s}, \phi_{s}\right) \text {. }
$$

However, the resulting harmonic coefficients in (2) are not accurate for commercial loudspeakers used in real scenario as they are non-ideal speakers. Thus, the problem addressed is to propose and compare various acoustic models to model the soundfield coefficients $\alpha_{n m}(k)$ in (2) due to a real commercial loudspeaker by a limited number of measurements and predict the reproduced sound field over an extended area.

\section{ACOUSTIC SOURCE MODELS}

This section discusses the proposed equivalent source models of plane wave, point source and the mixed source model in details, respectively.

\subsection{Plane wave modeling}

Suppose we can represent an equivalent soundfield due to a loudspeaker by a finite number of planes waves arriving from an equiangular grid over all 3D directions,

$$
P(\boldsymbol{x}, k) \approx \sum_{l=1}^{L} s\left(\hat{\boldsymbol{y}}_{l}, k\right) e^{i k \hat{\boldsymbol{y}}_{l} \cdot \boldsymbol{x}}
$$

where $s(\hat{\boldsymbol{y}}, k)$ is the driving signal of the plane wave arriving from the direction $\hat{\boldsymbol{y}}$. Using Gegenbauer expansion [19], we can write its decomposition in modal domain,

$$
e^{i k \hat{\boldsymbol{y}} \cdot \boldsymbol{x}}=\sum_{n=0}^{N} \sum_{m=-n}^{n} 4 \pi i^{n} Y_{n m}^{*}(\hat{\boldsymbol{y}}) j_{n}(k r) Y_{n m}(\theta, \phi) .
$$

By substituting (4) into (3) and equaling to (1), the equivalent harmonic coefficients $\alpha_{n m}(k)$ can be expressed as,

$$
\alpha_{n m}(k)=\sum_{l=1}^{L} 4 \pi i^{n} Y_{n m}^{*}\left(\hat{\boldsymbol{y}}_{l}\right) s\left(\hat{\boldsymbol{y}}_{l}, k\right) .
$$

which relates the spherical harmonic coefficients $\alpha_{n m}(k)$ that characterise the loudspeaker to an equivalent set of plane wave weights $s\left(\hat{\boldsymbol{y}}_{l}, k\right), l=1 . ., L$. Finally, we can write (5) in matrix form as,

$$
\boldsymbol{\alpha}=\boldsymbol{H}_{p w} \boldsymbol{s}_{p w}
$$

where $\boldsymbol{\alpha}=\left[\alpha_{00}, . ., \alpha_{N N}\right]^{T}, \boldsymbol{s}_{p w}=\left[s\left(\hat{\boldsymbol{y}}_{1}, k\right), . ., s\left(\hat{\boldsymbol{y}}_{L}, k\right)\right]^{T}$ and

$$
\boldsymbol{H}_{p w}=4 \pi i^{n}\left[\begin{array}{ccc}
Y_{00}^{*}\left(\hat{\boldsymbol{y}}_{l}\right) & \cdots & Y_{00}^{*}\left(\hat{\boldsymbol{y}}_{L}\right) \\
\cdots & \cdots & \cdots \\
Y_{N N}^{*}\left(\hat{\boldsymbol{y}}_{l}\right) & \cdots & Y_{N N}^{*}\left(\hat{\boldsymbol{y}}_{L}\right)
\end{array}\right]
$$

\subsection{Point source modeling}

Similarly, we propose to employ $M$ discrete point sources that lie on the surface of a sphere with radius of $r_{p}$ to realize the modeling by approximating the recorded sound pressure,

$$
P(\boldsymbol{x}, k) \approx \sum_{m=1}^{M} s\left(\hat{\boldsymbol{y}}_{m}, k\right) e^{i k\left\|\hat{\boldsymbol{y}}_{m}-\boldsymbol{x}\right\|_{2}} /\left\|\hat{\boldsymbol{y}}_{m}-\boldsymbol{x}\right\|_{2} .
$$

Various setting of radius $r_{p}$ makes a difference for the performance and its impact will be investigated in experiments. With Gegenbauer expansion [19], it can be decomposed as,

$$
\frac{e^{i k\|\hat{\boldsymbol{y}}-\boldsymbol{x}\|_{2}}}{\|\hat{\boldsymbol{y}}-\boldsymbol{x}\|_{2}}=\sum_{n=0}^{N} \sum_{m=-n}^{n} 4 \pi i k h_{n}\left(k r_{p}\right) Y_{n m}^{*}(\hat{\boldsymbol{y}}) j_{n}(k r) Y_{n m}(\theta, \phi) \text {. }
$$

By substituting (9) into (8) and equaling to (1), we obtain,

$$
\alpha_{n m}(k)=\sum_{m=1}^{M} 4 \pi i k h_{n}\left(k r_{p}\right) Y_{n m}^{*}\left(\hat{\boldsymbol{y}}_{m}\right) s\left(\hat{\boldsymbol{y}}_{m}, k\right) .
$$

Represent (10) in matrix form as,

$$
\boldsymbol{\alpha}=\boldsymbol{H}_{p s} \boldsymbol{s}_{p s}
$$

where $\boldsymbol{\alpha}=\left[\alpha_{00}, . ., \alpha_{N N}\right]^{T}, \boldsymbol{s}_{p s}=\left[s\left(\hat{\boldsymbol{y}}_{1}, k\right), . ., s\left(\hat{\boldsymbol{y}}_{M}, k\right)\right]^{T}$ and

$$
\boldsymbol{H}_{p s}=4 \pi i k h_{n}\left(k r_{s}\right)\left[\begin{array}{ccc}
Y_{00}^{*}\left(\hat{\boldsymbol{y}}_{1}\right) & \cdots & Y_{00}^{*}\left(\hat{\boldsymbol{y}}_{M}\right) \\
\ldots & \cdots & \ldots \\
Y_{N N}^{*}\left(\hat{\boldsymbol{y}}_{1}\right) & \cdots & Y_{N N}^{*}\left(\hat{\boldsymbol{y}}_{M}\right)
\end{array}\right]
$$

\subsection{Mixed source modeling}

This subsection proposes the mixed source model that exploits and combines both the plane wave and point source efficiently and models the recorded harmonic coefficients $\alpha_{n m}(k)$ in a joint way.

$$
\begin{aligned}
\alpha_{n m}(k)= & \sum_{l=1}^{L} 4 \pi i^{n} Y_{n m}^{*}\left(\hat{\boldsymbol{y}}_{l}\right) s\left(\hat{\boldsymbol{y}}_{l}, k\right) \\
& +\sum_{m=1}^{M} 4 \pi i k h_{n}\left(k r_{s}\right) Y_{n m}^{*}\left(\hat{\boldsymbol{y}}_{m}\right) s\left(\hat{\boldsymbol{y}}_{m}, k\right) .
\end{aligned}
$$

Combination of (6) and (11) leads to matrix form of (13),

$$
\boldsymbol{\alpha}=\boldsymbol{H}_{m s} \boldsymbol{s}_{m s}
$$

where $\boldsymbol{\alpha}=\left[\alpha_{00}, . ., \alpha_{N N}\right]^{T}, \boldsymbol{H}_{m s}=\left[\boldsymbol{H}_{p w} \boldsymbol{H}_{p s}\right]$ and $\boldsymbol{s}_{m s}=$ $\left[\boldsymbol{s}_{p w} \boldsymbol{s}_{p s}\right]^{T}$.

To combine the two parts fairly, grid of directions for the plane wave and point source shall be set by the same manner so that $L=M$. Moreover, for the sake of fair possibility to be selected, the amplitude of the plane wave and point source from the same direction $\hat{\boldsymbol{y}}$ ought to be equal. Therefore, the radius $r_{s}$ of point source within the mixed model is set for each frequency that follows $\left|k h_{n}\left(k r_{s}\right)\right|=1$ meter.

\section{SPARSE DECOMPOSITION}

This section attempts to seek for optimized or desired solutions for the acoustic models formulated in section 3 . Given (6), (11) and (14), traditional least square methods can provide accurate solutions while it has a tendency to spread the components of $\boldsymbol{\alpha}$ among a large number of source candidates in $\boldsymbol{H}$. Since we mainly consider the sound field generated by a single loudspeaker, we are to exploit the spatial sparsity feature by adding a sparse constraint using $\ell_{p}$ norm to the vector of driving signal $s$ shown in (15) as it manages to accomplish the modeling using only a small number of source candidates.

$$
\min _{\boldsymbol{s}}\|\boldsymbol{s}\|_{p}^{p} \text {, s.t. } \boldsymbol{H} \boldsymbol{s}=\boldsymbol{\alpha} .
$$

This paper investigates three effective sparse algorithms, e.g. IRLS, MP and LASSO, to solve the sparse problem in (15) and introduce each of them in brief. Note that all the subscripts in this section are abandoned for the sake of generality. IRLS; this algorithm [20,21] replaces the $\ell_{p}$ norm objective function in (15) by a form of weighted $\ell_{2}$ norm.

$$
\min _{\boldsymbol{s}} \sum_{i=1}^{M} \boldsymbol{w}_{i} \boldsymbol{s}_{i}^{2}, \text { s.t. } \boldsymbol{H} \boldsymbol{s}=\boldsymbol{\alpha}
$$

where $\boldsymbol{w}_{i}=\left|\boldsymbol{s}_{i}^{(m-1)}\right|^{p-2}$ and the driving signal for the next 


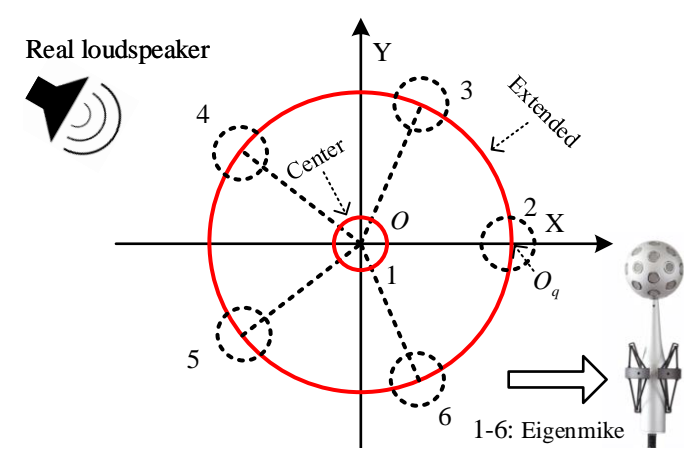

Fig. 1: Vertical view of the system setup for experiments. iterate $\boldsymbol{s}^{(m)}$ can be given explicitly,

$$
\boldsymbol{s}^{(m)}=Q_{m} \boldsymbol{H}^{T}\left(\boldsymbol{H} Q_{m} \boldsymbol{H}^{T}\right)^{-1} \boldsymbol{\alpha}
$$

where $Q_{m}$ is diagonal matrix with entries $1 / \boldsymbol{w}_{i}=\left|\boldsymbol{s}_{i}^{(m-1)}\right|^{2-p}$. MP; with a proper initialization, the MP conducts in iterative and greedy procedures to select the $m$-th column that has maximally inner product with current residual $R^{m} \boldsymbol{\alpha}$.

$$
\boldsymbol{h}^{(m)}=\arg \max _{\boldsymbol{h} \in \boldsymbol{H}}\left|\boldsymbol{h}^{T}\left(R^{m} \boldsymbol{\alpha}\right)\right|
$$

whose corresponding driving signal is calculated by,

$$
\mathbf{s}^{(m)}=\left|\left(\boldsymbol{h}^{(m)}\right)^{T} R^{m} \boldsymbol{\alpha}\right| .
$$

Above procedures comes to stop after enough number of iterations or when the residual $R^{(m)} \boldsymbol{\alpha}$ is close to zero [22].

LASSO; it reformulates (15) by combining the objective function and sparse constraint into a united expression.

$$
\boldsymbol{s}=\arg \min _{\boldsymbol{s}}\|\boldsymbol{\alpha}-\boldsymbol{H} \boldsymbol{s}\|_{2}^{2}+\lambda\|\boldsymbol{s}\|_{1} .
$$

The parameter $\lambda$ controls the extent of sparsity for vector $\mathbf{s}$. An optimal variable selection of LASSO for (20) can be realized by the coordinate descent algorithms [23].

\section{VALIDATION OF THE PROPOSED MODELS}

Intuitively, a successful theoretical model of the loudspeaker shall enable the ability to predict its incident field outside of the original measurements. Therefore, for validations, we use the proposed models to simulate the soundfield over an extended area and compare that to the real recordings. To make it, we design a practical measuring setup using direct measurements recorded by Eigenmike, which is demonstrated in Fig. 1 and note that the speaker symbolizes a real commercial loudspeaker. The small red circle of radius $4.2 \mathrm{~cm}$ at the center of Fig. 1, represents the Eigenmike recording used for modeling while the remained 5 Eigenmikes placed along the boundary of larger red circle with a radius of $0.3 \mathrm{~m}$ are employed for validations.

$$
\operatorname{Error}_{P}(k)=10 \log _{10}\left(\frac{\sum_{q=1}^{Q}\left|P^{\mathrm{rec}}\left(\theta_{q}, \phi_{q}, k\right)-P^{\mathrm{pre}}\left(\theta_{q}, \phi_{q}, k\right)\right|^{2}}{\sum_{q=1}^{Q}\left|P^{\mathrm{rec}}\left(\theta_{q}, \phi_{q}, k\right)\right|^{2}}\right)
$$

Assuming stationary conditions, we propose to emulate only one single Eigenmike moving along a horizontal circle to record the sound field for validations. The strategy to move the Eigenmike separately provides at least two benefits: the recording can be accomplished by one single Eigenmike so that the hardware costs can be reduced dramatically, and secondly it avoids perturbations of the scattering effects when to place several Eigenmikes around. For each model, the sound
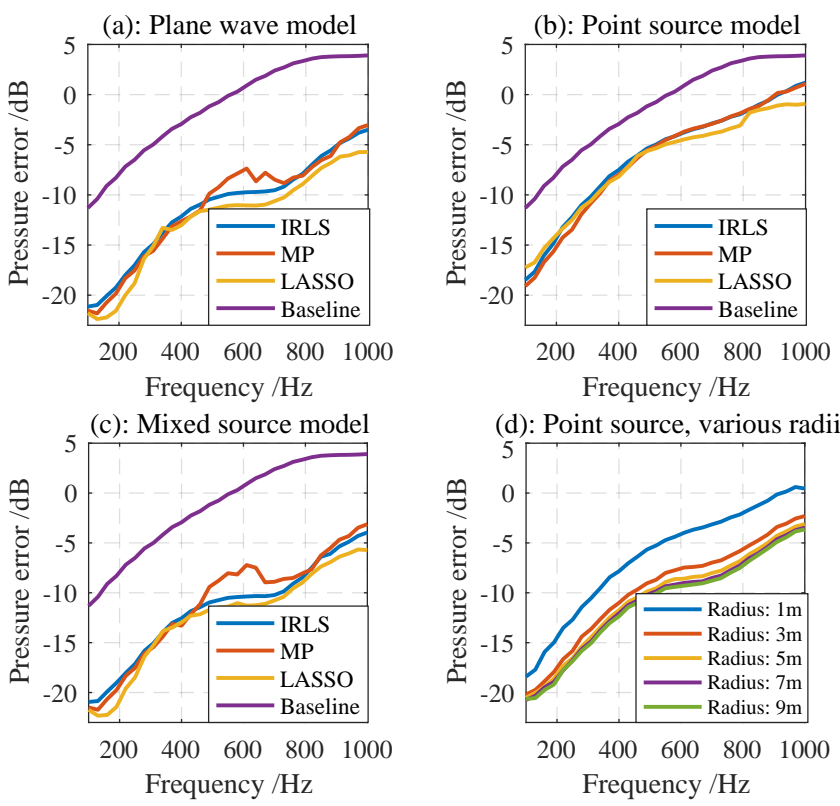

Fig. 2: Sound pressure errors for all acoustic models

pressure over the 5 Eigenmikes (160 channels in total) beyond the center can be approximated or simulated by the selected source candidates and their driving signal. To analyze the accuracy, the prediction errors over the extended area in terms of soundfield pressure are computed as (21) shows, in which the $P^{\text {rec }}(\cdot)$ and $P^{\text {pre }}(\cdot)$ represents the recorded and predicted sound pressure for each channel, respectively.

However, single metric of numerical sound pressure errors cannot fully evaluate or reflect the performance of the proposed sparsity exploited models. It is conceivable that a successful acoustic model exploiting sparsity shall enable the ability to select active candidates that encloses the direction of real loudspeaker. Therefore, apart from numerical error, spatial distributions of the active or selected source candidates with respect to direction of the real loudspeaker are investigated and taken into account for thorough evaluations. And such characteristics can be exhibited easily by plotting the distributions of the selected candidates as the Fig. 3 shows.

\section{EXPERIMENTS}

This section presents an experimental set up where a commercial loudspeaker's broadband response is recorded and modeled using the proposed equivalent source models. The performance of the proposed models is also analyzed using secondary measurements. Note that the real loudspeaker is part of an existing loudspeaker array of 30 units, which is used for spatial soundfield reproductions.

\subsection{Experimental setup}

The experiment's setup mainly consists of 4 stages. Firstly, we use an Eigenmike to record the soundfield due to an loudspeaker of interest. The loudspeaker is a single unit from the aforementioned sound reproduction system, and is located at $(r, \theta, \phi)=(1,0.55,0.62)$ with respect to the center of the listening area. The loudspeaker's incident sound field (only the direct path) is recorded at the origin using an Eigenmike as shown in Fig. 1. Secondly, the Eigenmike recordings are used to derive a set of theoretical source models using the proposed sparsity exploited methods. Thirdly, for validation of the proposed models outside of the original recording area, secondary soundfield recordings are obtained using a moving 

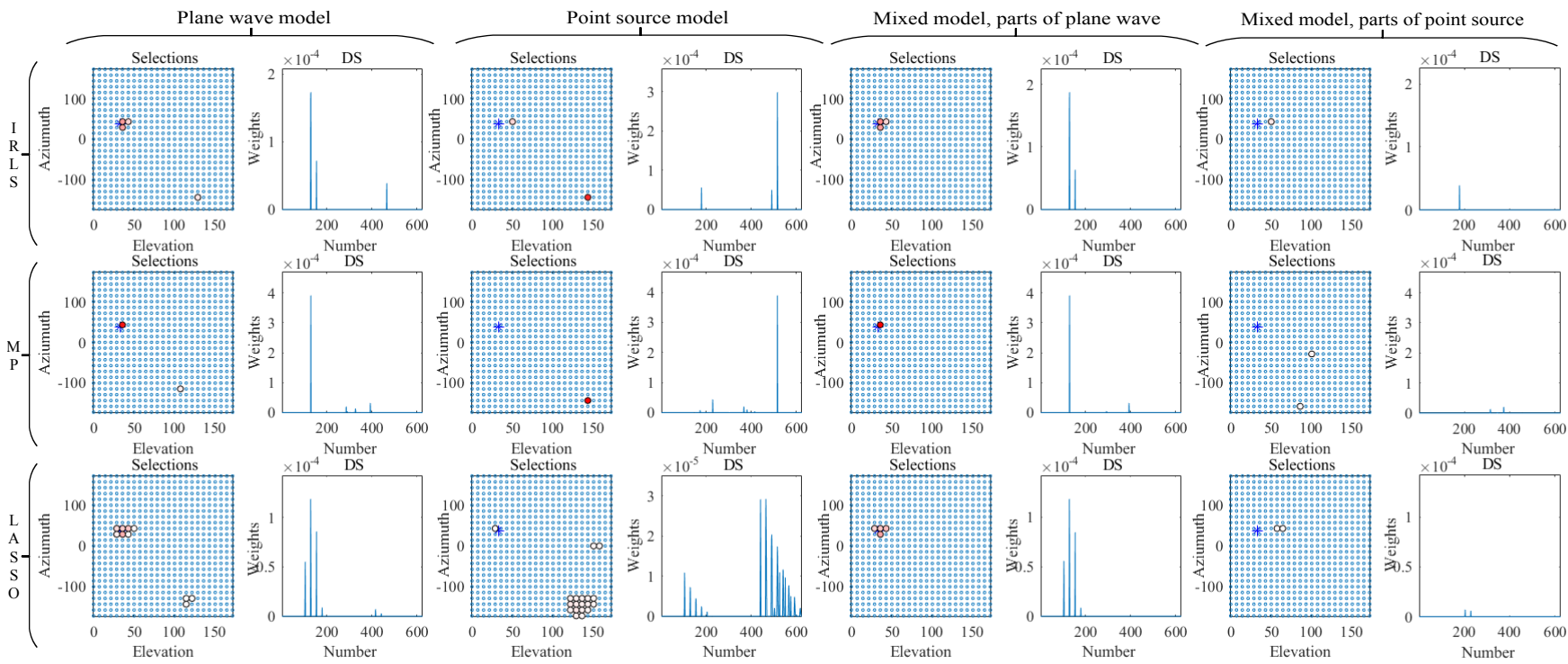

Fig. 3: Sparse distributions of selected candidates along with corresponding magnitude of driving signal (DS) for all models at $600 \mathrm{~Hz}$.

Eigenmike (see Fig. 1). Finally, we analyze the accuracy of the proposed methods using the metric given in (21). Furthermore, we also study the concept of sparsity exploited equivalent source models by analyzing the spatial distribution of the proposed source decompositions.

\subsection{Accuracy of the proposed models outside of the orig- inal recordings}

Here, we study the accuracy of the proposed models outside of the region where original recordings were taken. Note that we mainly consider frequencies below $1 \mathrm{kHz}$, because when soundfield reproduction accuracy is important, low frequencies are those mostly affected by loudspeaker directivity.

Sub-figures (a), (b) and (c) report the accuracy in terms of sound pressure errors for all acoustic models using the three sparse algorithms. Under low frequency conditions, the soundfield over the whole area in Fig. 1 may share certain similarities that makes it hard to distinguish the effectiveness of proposed modelings. Therefore, we calculate the differences of the sound pressure over the extended area with respect to the soundfield at the center and take it as a baseline.

Generally, each acoustic model using any sparse algorithm achieves satisfying performance, especially when the frequencies are below $500 \mathrm{~Hz}$. The performance decreases when the frequency increases in that the desired field is undersampled at higher frequencies. Among the sparse algorithms, the LASSO achieves with least numerical errors while it has a slow convergence rate and suffers from an expensive cost to determine the sparse parameter $\lambda$ in (20). The IRLS shows to be with more errors at low frequencies while outperforms the MP at higher frequency bins. For the acoustic models, the mixed acoustic model achieves competitive performance with a slight improvement when compared to the plane wave model. However, the point source model, with the same radius with that of mixed model, appears to be with more errors than the other two source models.

Figure 2(d) presents the mean sound pressure errors using all the three sparse algorithms for the point source model when they are placed at various radii. It demonstrates that the larger radius that lies further away from the sensors acts to be more like plane waves and leads to reduced modeling errors.

\subsection{Analysis of the spatial distribution of sparsity ex- ploited source models}

Figure 3 presents spatial distributions of selected source candidates for all proposed models using all sparse algorithms when $f=600 \mathrm{~Hz}$. Note that such distributions share similar profile over other frequency bins considered. For each sub-figure, the small circle lined in grid stands for the location of a candidate (625 in total) and the notation of $*$ in blue indicates the direction of real speaker. Magnitude of driving signal represents the degree of activity or importance for that candidate. The four double-columns marked by brackets correspond to distributions of plane wave model, point source model, the parts of plane wave and points source within the mixed source model, respectively. Three lines of figures from top to down employ IRLS, MP and LASSO, respectively.

Results verify that most of the active candidates selected by plane wave model lie around the direction of real speaker while the point source model fails. The mixed source model exhibits promising distributions in that the plane wave parts plays the leading role and a small amount of components originates from point source as well, which shall be kinds of signal components sharing a similar characteristics with point source. Considering the sparse solutions, the MP turns out to be the most sparse one due to the exponential decay of the residual error [22]. The IRLS, with less sound pressure errors than MP, provides satisfying sparse source distributions as well. Though the LASSO produces least numerical errors, it does not select an optimal sparse distribution due to that the global minimum of numerical error does not necessarily coincide with the optimal sparse solutions.

\section{CONCLUSION}

This paper has proposed several acoustic models to model inherent characteristics of commercial loudspeakers used in soundfield reproduction systems. Experimental results with promising performance have shown that both the plane wave and mixed source model possess an ability to characterize commercial speakers with acceptable accuracy. These models can be used in real sound reproduction systems to equalize for loudspeaker directivity, and the recording equipment could be any other suitable microphone array. 


\section{REFERENCES}

[1] W. Zhang, P. N. Samarasinghe, H. Chen, and T. D. Abhayapala, "Surround by sound: A review of spatial audio recording and reproduction," Applied Sciences, vol. 7, no. 5, pp. 532, 2017.

[2] M. Poletti, T. D. Abhayapala, and P. N. Samarasinghe, "Interior and exterior sound field control using two dimensional higher-order variable-directivity sources," The Journal of the Acoustical Society of America, vol. 131, no. 5, pp. 3814-3823, 2012.

[3] B. Rafaely, Fundamentals of spherical array processing, vol. 8, Springer, 2015.

[4] N. Radmanesh, I. S. Burnett, and B. D. Rao, "A lassols optimization with a frequency variable dictionary in a multizone sound system," IEEE/ACM Transactions on Audio, Speech and Language Processing, vol. 24, no. 3, pp. 583-593, 2016.

[5] J. Wenyu and W. B. Kleijn, "Theory and design of multizone soundfield reproduction using sparse methods," IEEE/ACM Transactions on Audio, Speech, and Language Processing, vol. 23, no. 12, pp. 2343-2355, 2015.

[6] H. Hacihabiboglu, E. De S., Z. Cvetkovic, J. Johnston, and J. O Smith III, "Perceptual spatial audio recording, simulation, and rendering: An overview of spatial-audio techniques based on psychoacoustics," IEEE Signal Processing Magazine, vol. 34, no. 3, pp. 36-54, 2017.

[7] D. B. Ward and T. D. Abhayapala, "Reproduction of a plane-wave sound field using an array of loudspeakers," IEEE Transactions on speech and audio processing, vol. 9, no. 6, pp. 697-707, 2001.

[8] P. N. Samarasinghe, M. A. Poletti, S. M. A. Salehin, T. D. Abhayapala, and F. M. Fazi, "3d soundfield reproduction using higher order loudspeakers," in Proc. IEEE International Conference on Acoustics, Speech and Signal Processing (ICASSP), Vancouver, Canada, 2013, pp. 306-310.

[9] A. H. Moore, M. Brookes, and P. A. Naylor, "Robust spherical harmonic domain interpolation of spatially sampled array manifolds," in Proc. IEEE International Conference on Acoustics, Speech and Signal Processing (ICASSP), New Orleans, USA, 2017, pp. 521525.

[10] P. N. Samarasinghe, T. D. Abhayapala, and H. C. Chen, "Estimating the direct-to-reverberant energy ratio using a spherical harmonics-based spatial correlation model," IEEE/ACM Transactions on Audio Speech and Language Processing, vol. 25, no. 2, pp. 310-319, 2017.

[11] A. J. Berkhout, D. de Vries, and P. Vogel, "Acoustic control by wave field synthesis," The Journal of the Acoustical Society of America, vol. 93, no. 5, pp. 2764-2778, 1993.
[12] S. Spors and J. Ahrens, "A comparison of wave field synthesis and higher-order ambisonics with respect to physical properties and spatial sampling," in Audio Engineering Society Convention 125, 2008.

[13] J. Ahrens, Applications of Sound Field Synthesis, pp. 175-272, Springer, 2012.

[14] S. Spors, H. Wierstorf, A. Raake, M. Melchior, F.and Frank, and F. Zotter, "Spatial sound with loudspeakers and its perception: A review of the current state," Proceedings of the IEEE, vol. 101, no. 9, pp. 1920-1938, 2013.

[15] Y. G. Hu, P. N. Samarasinghe, G. Dickins, and T. D. Abhayapala, "Modeling the interior response of real loudspeakers with finite measurements," in Proc. IEEE International Workshop on Acoustic Signal Enhancement (IWAENC), Tokyo, Japan, 2018.

[16] N. Murata, S. Koyama, N. Takamune, and H. Saruwatari, "Sparse representation using multidimensional mixed-norm penalty with application to sound field decomposition," IEEE Transactions on Signal Processing, 2018.

[17] E. G. Williams, Fourier acoustics: sound radiation and nearfield acoustical holography, Academic press, 1999.

[18] H. Khalilian, I. V. Bajic, and R. G. Vaughan, "Comparison of loudspeaker placement methods for sound field reproduction," IEEE-Acm Transactions on Audio Speech and Language Processing, vol. 24, no. 8, pp. 1364-1379, 2016.

[19] M. Abramowitz and I. A. Stegun, Handbook of mathematical functions: with formulas, graphs, and mathematical tables, vol. 55, Courier Corporation, 1964.

[20] R. Chartrand and Yin Wotao, "Iteratively reweighted algorithms for compressive sensing," in Proc. IEEE International Conference on Acoustics, Speech and Signal Processing (ICASSP), Las Vegas, USA, 2008, pp. 38693872.

[21] Y. Maeno, Y. Mitsufuji, and T. D. Abhayapala, "Mode domain spatial active noise control using sparse signal representation," in Proc. IEEE International Conference on Acoustics, Speech and Signal Processing (ICASSP), Alberta, Canada, 2018.

[22] S. G. Mallat and Z. F. Zhang, "Matching pursuits with time-frequency dictionaries," IEEE Transactions on signal processing, vol. 41, no. 12, pp. 3397-3415, 1993.

[23] T. T. Wu and K. Lange, "Coordinate descent algorithms for lasso penalized regression," The Annals of Applied Statistics, pp. 224-244, 2008. 